Pacific

Journal of

Mathematics

\title{
COMPUTING THE TUTTE POLYNOMIAL OF A HYPERPLANE ARRAGEMENT
}

FEDERICO ARDILA 


\title{
COMPUTING THE TUTTE POLYNOMIAL OF A HYPERPLANE ARRAGEMENT
}

\author{
FEDERICO ARDILA
}

\begin{abstract}
We define and study the Tutte polynomial of a hyperplane arrangement. We introduce a method for computing the Tutte polynomial by solving a related enumerative problem. As a consequence, we obtain new formulas for the generating functions enumerating alternating trees, labelled trees, semiorders and Dyck paths.
\end{abstract}

\section{Introduction}

Much work has been devoted in recent years to studying hyperplane arrangements and, in particular, their characteristic polynomials. The polynomial $\chi_{\mathscr{A}}(q)$ is a very powerful invariant of the arrangement $\mathscr{A}$; it arises very naturally in many different contexts. Two of the many important and beautiful results about the characteristic polynomial of an arrangement are the following.

Theorem 1.1 [Zaslavsky 1975]. Let $\mathscr{A}$ be a hyperplane arrangement in $\mathbb{R}^{n}$. The number of regions into which $A$ dissects $\mathbb{R}^{n}$ is equal to $(-1)^{n} \chi_{\mathscr{A}}(-1)$. The number of regions which are relatively bounded is equal to $(-1)^{n} \chi_{\mathscr{A}}(1)$.

Theorem 1.2 [Orlik and Solomon 1980]. Let $A$ be a hyperplane arrangement in $\mathbb{C}^{n}$, and let $M_{\mathscr{A}}=\mathbb{C}^{n}-\bigcup_{H \in \mathscr{A}} H$ be its complement. Then the Poincaré polynomial of the cohomology ring of $M_{\mathscr{A}}$ is given by:

$$
\sum_{k \geq 0} \operatorname{rank} H^{k}\left(M_{\mathscr{A}}, \mathbb{Z}\right) q^{k}=(-q)^{n} \chi_{\mathscr{A}}(-1 / q) .
$$

Several authors have worked on computing the characteristic polynomials of specific hyperplane arrangements. This work has led to some very nice enumerative results; see for example [Athanasiadis 1996; Postnikov and Stanley 2000].

Somewhat surprisingly, nothing has been said about the Tutte polynomial of a hyperplane arrangement. Graphs and matroids have a Tutte polynomial associated with them, which generalizes the characteristic polynomial and arises naturally

MSC2000: primary 52C35; secondary 05B35, 05A15.

Keywords: hyperplane arrangement, Tutte polynomial, finite field method. 
in numerous enumerative problems in both areas. Many interesting invariants of graphs and matroids can be computed immediately from this polynomial.

The present paper aims to define and investigate the Tutte polynomial of a hyperplane arrangement. This paper is devoted to purely enumerative questions, particularly the computation of Tutte polynomials of specific arrangements. We addressed the matroid-theoretic aspects of this investigation in [Ardila 2004].

In Section 2 we introduce the basic notions of hyperplane arrangements that we will need. In Section 3 we define the Tutte polynomial of a hyperplane arrangement, and we present a finite field method for computing it. This is done in terms of the coboundary polynomial, a simple transformation of the Tutte polynomial. We recover several known results about the characteristic and Tutte polynomials of graphs and representable matroids, and derive other consequences of this method. In Section 4 we compute the Tutte polynomials of Coxeter arrangements, threshold arrangements, and generic deformations of the braid arrangement. In Section 5 we focus on a large family of deformations of the braid arrangement, where the computation of Tutte polynomials is related to the enumeration of classical combinatorial objects. As a consequence, we obtain several purely enumerative results about alternating trees, parking functions, semiorders, and Dyck paths.

\section{Hyperplane arrangements}

We recall some of the basic concepts of hyperplane arrangements. For a more thorough introduction, see [Orlik and Terao 1992] or [Stanley 2004].

Given a field $\mathbb{k}$ and a positive integer $n$, an affine hyperplane in $\mathbb{k}^{n}$ is an $(n-1)$ dimensional affine subspace of $\mathbb{k}^{n}$. If we fix a system of coordinates $x_{1}, \ldots, x_{n}$ on $\mathbb{k}^{n}$, a hyperplane can be seen as the set of points that satisfy a certain equation $c_{1} x_{1}+\cdots+c_{n} x_{n}=c$, where $c_{1}, \ldots, c_{n}, c \in \mathbb{k}$ and not all $c_{i}$ s are equal to 0 . A hyperplane arrangement $\mathscr{A}$ in $\mathbb{k}^{n}$ is a finite set of affine hyperplanes of $\mathbb{k}^{n}$. We will refer to hyperplane arrangements simply as arrangements. We will mostly be interested in arrangements in $\mathbb{R}^{n}$, but we will find it useful to work over other fields as well.

We will say that an arrangement $\mathscr{A}$ is central if the hyperplanes in $\mathscr{A}$ have a nonempty intersection. (Sometimes we will call an arrangement affine to emphasize that it does not need to be central.) Similarly, we will say that a subset (or subarrangement) $\mathscr{B} \subseteq \mathscr{A}$ of hyperplanes is central if the hyperplanes in $\mathscr{B}$ have a nonempty intersection.

The rank function $r_{\mathscr{A}}$ is defined for each central subset $\mathscr{B}$ by $r_{\mathscr{A}}(\mathscr{B})=n-$ $\operatorname{dim} \bigcap \Re$. This function can be extended to a function $r_{\mathscr{A}}: 2^{\mathscr{A}} \rightarrow \mathbb{N}$, by defining the rank of a noncentral subset $\mathscr{B}$ to be the largest rank of a central subset of $\mathscr{B}$. The rank of $\mathscr{A}$ is $r_{\mathscr{A}}(\mathscr{A})$, and it is denoted $r_{\mathscr{A}}$. 
Alternatively, if the hyperplane $H$ has defining equation $c_{1} x_{1}+\cdots+c_{n} x_{n}=c$, associate its normal vector $v=\left(c_{1}, \ldots, c_{n}\right)$ to it. Then define $r_{\mathscr{A}}\left(\left\{H_{1}, \ldots, H_{k}\right\}\right)$ to be the dimension of the span of the corresponding vectors $v_{1}, \ldots, v_{k}$ in $\mathbb{R}^{n}$. It is easy to see that these two definitions of the rank function agree. In particular, this means that the resulting function $r_{\mathscr{A}}: 2^{\mathscr{A}} \rightarrow \mathbb{N}$ is the rank function of a matroid. We will usually omit the subscripts when the underlying arrangement is clear, and simply write $r(\mathscr{B})$ and $r$ for $r_{\mathscr{A}}(\mathscr{B})$ and $r_{\mathscr{A}}$, respectively.

(Note that there is another natural way to extend $r_{\mathscr{A}}$ to the rank function of a matroid; see [Ardila 2004].)

The rank function gives us natural definitions of the usual concepts of matroid theory, such as independent sets, bases, flats, and circuits, in the context of hyperplane arrangements. All of this is done more naturally in the broader context of semimatroids in [Ardila 2004].

To each hyperplane arrangement $\mathscr{A}$ we assign a partially ordered set, called the intersection poset of $\mathscr{A}$ and denoted $L_{\mathscr{A}}$. It consists of the nonempty intersections $H_{i_{1}} \cap \cdots \cap H_{i_{k}}$, ordered by reverse inclusion. This poset is graded, with rank function $r\left(H_{i_{1}} \cap \cdots \cap H_{i_{k}}\right)=r_{\mathscr{A}}\left(\left\{H_{i_{1}}, \ldots, H_{i_{k}}\right\}\right)$, and a unique minimal element $\hat{0}=\mathbb{R}^{n}$. We will call two arrangements $\mathscr{A}_{1}$ and $\mathscr{A}_{2}$ combinatorially isomorphic or simply isomorphic, and write $\mathscr{A}_{1} \cong \mathscr{A}_{2}$, if $L_{\mathscr{A}_{1}} \cong L_{\mathscr{A}_{2}}$. Isomorphic arrangements may be defined over different fields.

The characteristic polynomial of $\mathscr{A}$ is

$$
\chi_{\mathscr{A}}(q)=\sum_{x \in L_{\mathscr{A}}} \mu(\hat{0}, x) q^{n-r(x)}
$$

where $\mu$ denotes the Möbius function of $L_{\mathscr{A}}$ [Stanley 1997, Section 3.7].

Let $\mathscr{A}$ be an arrangement and let $H$ be a hyperplane in $\mathscr{A}$. The arrangement $\mathscr{A}-\{H\}$ (or simply $\mathscr{A}-H$ ), obtained by removing $H$ from the arrangement, is called the deletion of $H$ in $\mathscr{A}$. It is an arrangement in $\mathbb{R}^{n}$. The arrangement $\mathscr{A} / H=$ $\left\{H^{\prime} \cap H \mid H^{\prime} \in \mathscr{A}-H, H^{\prime} \cap H \neq \varnothing\right\}$, consisting of the intersections of the other hyperplanes with $H$, is called the contraction of $H$ in $\mathscr{A}$. It is an arrangement in $H$.

However, some technical difficulties can arise. In a hyperplane arrangement $\mathscr{A}$, contracting a hyperplane $H$ may give us repeated hyperplanes $H_{1}$ and $H_{2}$ in the arrangement $\mathscr{A} / H$. Say we want to contract $H_{1}$ in $\mathscr{A} / H$. In passing to the contraction $(\mathscr{A} / H) / H_{1}$, the hyperplane $H_{2}$ of $\mathscr{A} / H$ becomes the "hyperplane" $H_{2} \cap H_{1}=H_{1}$ in the "arrangement" $(\mathscr{A} / H) / H_{1}$. But this is not a hyperplane in $H_{1}$.

Therefore, the class of hyperplane arrangements, as defined, is not closed under deletion and contraction. This is problematic when we want to mirror matroidtheoretic results in this context. There is an artificial solution to this problem: we can consider multisets $\left\{H_{1}, \ldots, H_{k}\right\}$ of subspaces of a vector space $V$, where each 
$H_{i}$ has $\operatorname{dimension} \operatorname{dim} V-1$ or $\operatorname{dim} V$. In other words, we allow repeated hyperplanes, and we allow the full space $V$ to be regarded as a "hyperplane", mirroring a loop of a matroid. This class of objects is closed under deletion and contraction, but it is somewhat awkward to work with. A better solution is to think of arrangements as members of the class of semimatroids: a class that is also closed under deletion and contraction, and is more natural matroid-theoretically. We develop this point of view in [Ardila 2004]. However, such issues will be irrelevant in this paper, which focuses on purely enumerative aspects of arrangements.

\section{Computing the Tutte polynomial}

Athanasiadis [1996] introduced a powerful method for computing the characteristic polynomial of a subspace arrangement, based on ideas of Crapo and Rota [1970]. He reduced the computation of characteristic polynomials to an enumeration problem in a vector space over a finite field. He used this method to compute explicitly the characteristic polynomial of several families of hyperplane arrangements, obtaining very nice enumerative results. As should be expected, this method only works when the equations defining the hyperplanes of the arrangement have integer (or rational) coefficients. Such an arrangement will be called a $\mathbb{Z}$-arrangement.

Reiner discovered an elegant interpretation for the Tutte polynomial $T_{M}$ of a representable matroid $M$ (Equation (3) in [1999]), and asked whether it could be used to compute $T_{M}$ for some nontrivial families of matroids. Compared to all the work that has been done on computing characteristic polynomials explicitly, virtually nothing is known about computing Tutte polynomials.

Our Theorem 3.3 below gives a new method for computing Tutte polynomials of hyperplane arrangements. Our approach does not use Reiner's result; it is closer to Athanasiadis' method. After proving the theorem, we present some of its consequences, using it in Section 4 to compute explicitly the Tutte polynomials of several families of arrangements.

\section{The Tutte and coboundary polynomials.}

Definition 3.1. The Tutte polynomial of a hyperplane arrangement $\mathscr{A}$ is

$$
T_{\mathscr{A}}(x, y)=\sum_{\substack{\mathscr{B} \subseteq \mathscr{A} \\ \text { central }}}(x-1)^{r-r(\mathscr{B})}(y-1)^{|\mathscr{B}|-r(\mathscr{B})},
$$

where the sum is over all central subsets $\mathscr{B} \subseteq \mathscr{A}$.

If $\mathscr{A}$ is central and $M(\mathscr{A})$ is its associated matroid, this definition coincides with the usual definition of the Tutte polynomial of the matroid $M(\mathscr{A})$.

It will be useful for us to consider a simple transformation of the Tutte polynomial, first considered by Crapo [1969] in the context of matroids. 
Definition 3.2. The coboundary polynomial $\bar{\chi}_{\mathscr{A}}(q, t)$ of an arrangement $\mathscr{A}$ is

$$
\bar{\chi}_{\mathscr{A}}(q, t)=\sum_{\substack{\Re \subseteq A \\ \text { central }}} q^{r-r(\mathscr{B})}(t-1)^{|\mathscr{B}|} .
$$

It is easy to check that

$$
\bar{\chi}_{\mathscr{A}}(q, t)=(t-1)^{r} T_{\mathscr{A}}\left(\frac{q+t-1}{t-1}, t\right)
$$

and

$$
T_{\mathscr{A}}(x, y)=\frac{1}{(y-1)^{r}} \bar{\chi}_{\mathscr{A}}((x-1)(y-1), y) .
$$

Therefore, computing the coboundary polynomial of an arrangement is essentially equivalent to computing its Tutte polynomial. The results in this paper can be presented more elegantly in terms of the coboundary polynomial.

Also, recall Whitney's theorem [Stanley 2004, Theorem 2.4], which states that

$$
\chi_{\mathscr{A}}(q)=\sum_{\substack{\mathscr{B} \subseteq \mathscr{A} \\ \text { central }}}(-1)^{|\mathscr{B}|} q^{n-r(\mathscr{B})} .
$$

This allows us to express the characteristic polynomial in terms of the coboundary polynomial:

$$
\chi_{\mathscr{A}}(q)=q^{n-r} \bar{\chi}_{\mathscr{A}}(q, 0)
$$

The finite field method. Let $\mathscr{A}$ be a $\mathbb{Z}$-arrangement in $\mathbb{R}^{n}$, and let $q$ be a prime power. The arrangement $\mathscr{A}$ induces an arrangement $\mathscr{A}_{q}$ in the vector space $\mathbb{F}_{q}^{n}$. If we consider the equations defining the hyperplanes of $\mathscr{A}$, and regard them as equations over $\mathbb{F}_{q}$, they define the hyperplanes of $\mathscr{A}_{q}$.

Say that $\mathscr{A}$ reduces correctly over $\mathbb{F}_{q}$ if the arrangements $\mathscr{A}_{\text {and }} \mathscr{A}_{q}$ are isomorphic. This does not always happen; sometimes the hyperplanes of $\mathscr{A}$ do not even become hyperplanes in $\mathscr{A}_{q}$. For example, the hyperplane $2 x+2 y=1$ in $\mathbb{R}^{2}$ becomes the empty "hyperplane" $0=1$ in $\mathbb{F}_{2}^{2}$. Sometimes independence is not preserved. For example, the independent hyperplanes $2 x+y=0$ and $y=0$ in $\mathbb{R}^{2}$ become the same hyperplane in $\mathbb{F}_{2}^{2}$.

However, if $q$ is a power of a large enough prime, $\mathscr{A}$ will reduce correctly over $\mathbb{F}_{q}$. To have $\mathscr{A} \cong \mathscr{A}_{q}$, we need central and independent subarrangements to be preserved. Cramer's rule lets us rephrase these conditions, in terms of certain determinants (formed by the coefficients of the hyperplanes in $\mathscr{A}$ ) being zero or nonzero. If $q$ is a power of a prime which is larger than all these determinants, we will guarantee that $\mathscr{A}$ reduces correctly over $\mathbb{F}_{q}$. 
Theorem 3.3. Let $A$ be a $\mathbb{Z}$-arrangement in $\mathbb{R}^{n}$. Let $q$ be a power of a large enough prime, and let $\mathscr{A}_{q}$ be the induced arrangement in $\mathbb{F}_{q}^{n}$. Then

$$
q^{n-r} \bar{\chi}_{\mathscr{A}}(q, t)=\sum_{p \in \mathbb{F}_{q}^{n}} t^{h(p)}
$$

where $h(p)$ denotes the number of hyperplanes of $A_{q}$ that $p$ lies on.

Proof. Let $q$ be a power of a large enough prime, so that $\mathscr{A}$ reduces correctly over $\mathbb{F}_{q}$. For each $\mathscr{B} \subseteq \mathscr{A}$, let $\mathscr{B}_{q}$ be the subarrangement of $\mathscr{A}_{q}$ induced by it. For each $p \in \mathbb{F}_{q}^{n}$, let $H(p)$ be the set of hyperplanes of $\mathscr{A}_{q}$ that $p$ lies on. From (3-2) we have

$$
\begin{aligned}
q^{n-r} \bar{\chi}_{\mathscr{A}}(q, t) & =\sum_{\substack{\mathscr{B} \subseteq \mathscr{A} \\
\text { central }}} q^{n-r(\mathscr{B})}(t-1)^{|\mathscr{B}|}=\sum_{\substack{\mathscr{B} \subseteq \mathscr{A} \\
\text { central }}} q^{\operatorname{dim} \cap \Re}(t-1)^{|\mathscr{B}|} \\
& =\sum_{\substack{\mathscr{B}_{q} \subseteq \mathscr{A}_{q} \\
\text { central }}}\left|\cap \mathscr{B}_{q}\right|(t-1)^{\left|\mathscr{B}_{q}\right|}=\sum_{\substack{\mathscr{B}_{q} \subseteq \mathscr{A}_{q} \\
\text { central }}} \sum_{p \in \cap \mathscr{\Re}_{q}}(t-1)^{\left|\mathscr{P}_{q}\right|} \\
& =\sum_{p \in \mathbb{F}_{q}^{n}} \sum_{\mathscr{B}_{q} \subseteq H(p)}(t-1)^{\left|\mathscr{B}_{q}\right|}=\sum_{p \in \mathbb{F}_{q}^{n}}(1+(t-1))^{h(p)},
\end{aligned}
$$

as desired.

In principle, Theorem 3.3 only computes $\bar{\chi}_{\mathscr{A}}(q, t)$ when $q$ is a power of a large enough prime. In practice, however, when we compute the right-hand side of (3-3) for large prime powers $q$, we will get a polynomial function in $q$ and $t$. Since the left-hand side is also a polynomial, these two polynomials must be equal.

Theorem 3.3 reduces the computation of coboundary polynomials (and hence Tutte polynomials) to enumerating points in the finite vector space $\mathbb{F}_{q}^{n}$, according to a certain statistic. This method can be extremely useful when the hyperplanes of the arrangement are defined by simple equations. We illustrate this in Section 4.

We remark that Theorem 3.3 was also obtained by Welsh and Whittle [1999, Theorem 7.1]. Also, since the characteristic polynomial of $\mathscr{A}$ is given by $\chi_{\mathscr{A}}(q)=$ $q^{n-r} \bar{\chi}_{\mathscr{A}}(q, 0)$, the special case $t=0$ is the following result:

Theorem 3.4 [Athanasiadis 1996, Theorem 2.2]. If $A$ and $q$ are as in Theorem 3.3, then $\chi_{\mathscr{A}}(q)$ is the number of points in $\mathbb{F}_{q}^{n}$ which are not on any of the hyperplanes of $\mathscr{A}_{q}$; that is,

$$
\chi_{\mathscr{A}}(q)=\left|\mathbb{F}_{q}^{n}-\mathscr{A}_{q}\right|
$$

Special cases and applications. We now show how the finite field method of Theorem 3.3 can be used to give straightforward proofs of four known facts (Theorems 3.5, 3.6, 3.7 , 3.8) and an apparently new result (Theorem 3.9) on Tutte polynomials. 
Colorings of graphs. Given a graph $G$ on $[n]$, we associate to it an arrangement $\mathscr{A}_{G}$ in $\mathbb{R}^{n}$. It consists of the hyperplanes $x_{i}=x_{j}$, for all $1 \leq i<j \leq n$ such that $i j$ is an edge in the graph $G$. It is easy to see that the matroid of $\mathscr{A}_{G}$ is the cycle matroid of $G$, so $T_{\mathscr{A}_{G}}(x, y)$ coincides with the (graph-theoretic) Tutte polynomial $T_{G}(x, y)$. We can define the coboundary polynomial for a graph as we did for arrangements, and then $\bar{\chi}_{G}(q, t)=\bar{\chi}_{\mathscr{A}_{G}}(q, t)$ also.

We shall now interpret Theorem 3.3 in this framework. It is easy to see that the rank of $\mathscr{A}_{G}$ is equal to $n-c$, where $c$ is the number of connected components of $G$. Therefore the left-hand side of (3-3) is $q^{c} \bar{\chi}_{G}(q, t)$ in this case.

To interpret the right-hand side, notice that each point $p \in \mathbb{F}_{q}^{n}$ corresponds to a $q$-coloring of the vertices of $G$. The point $p=\left(p_{1}, \ldots, p_{n}\right)$ will correspond to the coloring $\kappa_{p}$ of $G$ which assigns color $p_{i}$ to vertex $i$. A hyperplane $x_{i}=x_{j}$ contains $p$ when $p_{i}=p_{j}$. This happens precisely when edge $i j$ is monochromatic in $\kappa_{p}$; that is, when its two ends have the same color. Therefore, applying Theorem 3.3 to the arrangement $\mathscr{A}_{G}$, we recover the following known result:

Theorem 3.5 [Brylawski and Oxley 1992, Proposition 6.3.26]. Let G be a graph with c connected components. Then

$$
q^{c} \bar{\chi}_{G}(q, t)=\sum_{\substack{q-\text { colorings } \\ \kappa \text { of } G}} t^{\operatorname{mono}(\kappa)},
$$

where $\operatorname{mono}(\kappa)$ is the number of monochromatic edges in $\kappa$.

Linear codes. Given positive integers $n \geq r$, an $[n, r]$ linear code $C$ over $\mathbb{F}_{q}$ is an $r$-dimensional subspace of $\mathbb{F}_{q}^{n}$. A generator matrix for $C$ is an $r \times n$ matrix $U$ over $\mathbb{F}_{q}$, the rows of which form a basis for $C$. It is not difficult to see that the isomorphism class of the matroid on the columns of $U$ depends only on $C$. We shall denote the corresponding matroid $M_{C}$.

The elements of $C$ are called codewords. The weight $w(v)$ of a codeword is the cardinality of its support; that is, the number of nonzero coordinates of $v$. The codeweight polynomial of $C$ is

$$
A(C, q, t)=\sum_{v \in C} t^{w(v)} .
$$

The translation of Theorem 3.3 to this setting is the following.

Theorem 3.6 [Greene 1976]. For any linear code C over $\mathbb{F}_{q}$,

$$
A(C, q, t)=t^{n} \bar{\chi}_{M_{C}}\left(q, \frac{1}{t}\right) .
$$

Proof. Let $\mathscr{A}_{C}$ be the central arrangement corresponding to the columns of $U$. (We can call it $\mathscr{A}_{C}$ because, as stated above, its isomorphism class depends only on $C$.) 
This is a rank $r$ arrangement in $\mathbb{F}_{q}^{r}$ such that $\bar{\chi}_{M_{C}}\left(q, \frac{1}{t}\right)=\bar{\chi}_{\mathscr{A}_{C}}\left(q, \frac{1}{t}\right)$. Comparing (3-4) with Theorem 3.3, it remains to prove that

$$
\sum_{v \in C} t^{w(v)}=\sum_{p \in \mathbb{F}_{q}^{r}} t^{n-h(p)}
$$

To do this, consider the bijection $\phi: \mathbb{F}_{q}^{r} \rightarrow C$ determined by right multiplication by $U$. If $u_{1}, \ldots, u_{r}$ are the row vectors of $U$, then $\phi$ sends $p=\left(p_{1}, \ldots, p_{r}\right) \in \underset{q}{r}$ to the codeword $v_{p}=p_{1} u_{1}+\cdots+p_{r} u_{r} \in C$. For $1 \leq i \leq n, p$ lies on the hyperplane determined by the $i$ th column of $U$ if and only if the $i$ th coordinate of $v_{p}$ is equal to zero. Therefore $h(p)=n-w\left(v_{p}\right)$. This completes the proof.

Deletion-contraction. The point of view of Theorem 3.3 can be used to give a simple enumerative proof of the deletion-contraction formula for the Tutte polynomial of an arrangement. Once again, this formula is more natural in the context of semimatroids, as shown in [Ardila 2004]. For the moment, leaving matroid-theoretical issues aside, we only wish to present a special case of it as an application.

Theorem 3.7. Let $\mathscr{A}$ be a hyperplane arrangement, and let $H$ be a hyperplane in $\mathscr{A}$ such that $r_{\mathscr{A}}(\mathscr{A}-H)=r_{\mathscr{A}}$. Then $T_{\mathscr{A}}(x, y)=T_{\mathscr{A}-H}(x, y)+T_{\mathscr{A} / H}(x, y)$.

Proof. Because there will be several arrangements involved, let $h(\mathscr{B}, p)$ denote the number of hyperplanes in $\mathscr{P}_{q}$ that $p$ lies on. Then

$$
\begin{aligned}
q^{n-r} \bar{\chi}_{\mathscr{A}}(q, t) & =\sum_{p \in \mathbb{F}_{q}^{n}} t^{h(\mathscr{A}, p)}=\sum_{p \in \mathbb{F}_{q}^{n}-H} t^{h(\mathscr{A}, p)}+\sum_{p \in H} t^{h(\mathscr{A}, p)} \\
& =\sum_{p \in \mathbb{F}_{q}^{n}-H} t^{h(\mathscr{A}-H, p)}+\sum_{p \in H} t^{h(\mathscr{A}-H, p)+1} \\
& =\sum_{p \in \mathbb{F}_{q}^{n}} t^{h(\mathscr{A}-H, p)}+(t-1) \sum_{p \in H} t^{h(\mathscr{A}-H, p)} \\
& =q^{n-r} \bar{\chi}_{\mathscr{A}-H}(q, t)+(t-1) q^{(n-1)-(r-1)} \bar{\chi}_{\mathscr{A} / H}(q, t) .
\end{aligned}
$$

We conclude that $\bar{\chi}_{\mathscr{A}}(q, t)=\bar{\chi}_{\mathscr{A}-H}(q, t)+(t-1) \bar{\chi}_{\mathscr{A} / H}(q, t)$, which is equivalent to the deletion-contraction formula for Tutte polynomials.

A Möbius formula. The finite field method, when combined with the Möbius inversion formula for posets, naturally gives an alternative formula for the coboundary polynomial. This formula, in the context of matroids, is due to Crapo [1968].

Theorem 3.8. For an arrangement $\mathscr{A}$ and an affine subspace $x$ in the intersection poset $L_{\mathscr{A}}$, let $h(x)$ be the number of hyperplanes of $\mathscr{A}$ containing $x$. Then

$$
\bar{\chi}_{\mathscr{A}}(q, t)=\sum_{x \leq y \text { in } L_{\mathscr{A}}} \mu(x, y) q^{r-r(y)} t^{h(x)} .
$$


Proof. Consider the arrangement $\mathscr{A}$ restricted to $\mathbb{F}_{q}^{n}$, where $q$ is a power of a large enough prime, so that $\mathscr{A}$ reduces correctly over $\mathbb{F}_{q}$. Given $x \in L_{\mathscr{A}_{q}}$, let $P(x)$ be the set of points in $\mathbb{F}_{q}^{n}$ which are contained in $x$, and are not contained in any $y$ such that $y>x$ in $L_{\mathscr{A}_{q}}$. Then the set $x$ is partitioned by the sets $P(y)$ for $y \geq x$, so we have

$$
q^{\operatorname{dim} x}=|x|=\sum_{y \geq x}|P(y)| .
$$

By the Möbius inversion formula [Stanley 1997, Proposition 3.7.1] we have

$$
|P(x)|=\sum_{y \geq x} \mu(x, y) q^{\operatorname{dim} y} .
$$

Now, from Theorem 3.3 we know that

$q^{n-r} \bar{\chi}_{\mathscr{A}}(q, t)=\sum_{x \in L_{\mathscr{A}}} \sum_{p \in P(x)} t^{h(p)}=\sum_{x \in L_{\mathscr{A}}}|P(x)| t^{h(x)}=\sum_{x \leq y \text { in } L_{\mathscr{A}}} \mu(x, y) q^{n-r(y)} t^{h(x)}$,

as desired.

A probabilistic interpretation. In matroid reliability and percolation problems, one starts with a fixed matroid $M$. Each element of the ground set of $M$ has a certain probability of being deleted, independently of the other elements. One then asks for the probability that the retained elements satisfy a certain property. See [Brylawski and Oxley 1992, Section 6.3.E] for more on this subject. The following theorem is similar in spirit to these results, and it may be applied to the analogous questions concerning hyperplane arrangements.

Theorem 3.9. Let $\mathscr{A}$ be an arrangement and let $0 \leq t \leq 1$ be a real number. Let $\mathscr{B}$ be a random subarrangement of $\mathscr{A}$, obtained by independently removing each hyperplane from $A$ with probability $t$. Then the expected characteristic polynomial $\chi_{\mathscr{B}}(q)$ of $\mathscr{B}$ is $q^{n-r} \bar{\chi}_{\mathscr{A}}(q, t)$.

Proof. We have

$$
\begin{aligned}
E\left[\chi_{\mathscr{B}}(q)\right] & =\sum_{\mathscr{C} \subseteq \mathscr{A}} \operatorname{Pr}[\mathscr{B}=\mathscr{C}] \chi_{\mathscr{C}}(q)=\sum_{\mathscr{C} \subseteq \mathscr{A}} \operatorname{Pr}[\mathscr{B}=\mathscr{C}]\left|\mathbb{F}_{q}^{n}-\mathscr{C}_{q}\right| \\
& =\sum_{p \in \mathbb{F}_{q}^{n}} \sum_{\substack{\mathscr{C} \subseteq \mathscr{A} \\
p \notin \mathscr{C}_{q}}} \operatorname{Pr}[\mathscr{B}=\mathscr{C}],
\end{aligned}
$$

where in the second step we have used Theorem 3.4.

Recall that $H(p)$ denotes the set of hyperplanes in $\mathscr{A}_{q}$ containing $p$. Then

$$
E\left[\chi_{\mathscr{B}}(q)\right]=\sum_{p \in \mathbb{F}_{q}^{n}} \operatorname{Pr}\left[\mathscr{P}_{q} \cap H(p)=\varnothing\right]=\sum_{p \in \mathbb{F}_{q}^{n}} t^{h(p)},
$$

which is precisely what we wanted to show. 


\section{Computing coboundary polynomials}

In this section we use Theorem 3.3 to compute the coboundary polynomials of several families of arrangements. As remarked on page 4, this is essentially the same as computing their Tutte polynomials.

Coxeter arrangements. To illustrate how our finite field method works, we start by presenting some simple examples.

Let $\Phi$ be an irreducible crystallographic root system in $\mathbb{R}^{n}$, and let $W$ be its associated Weyl group. The Coxeter arrangement of type $W$ consists of the hyperplanes $(\alpha, x)=0$ for each $\alpha \in \Phi^{+}$, with the standard inner product on $\mathbb{R}^{n}$. See [Björner and Brenti 2005] or [Humphreys 1990] for an introduction to root systems and Weyl groups, and [Björner et al. 1993, Section 2.3] or [Orlik and Terao 1992, Chapter 6] for more information on Coxeter arrangements.

In this section we compute the coboundary polynomials of the Coxeter arrangements of type $A_{n}, B_{n}$ and $D_{n}$. (The arrangement of type $C_{n}$ is the same as the arrangement of type $B_{n}$.) The best way to state our results is to compute the exponential generating function for the coboundary polynomials of each family.

The following three theorems have never been stated explicitly in the literature in this form. Theorem 4.1 is equivalent to a result of Tutte [1954], who computed the Tutte polynomial of the complete graph. It is also an immediate consequence of a more general theorem of Stanley [1998a, (15)]. Theorems 4.2 and 4.3 are implicit in the work of Zaslavsky [1995].

Theorem 4.1. Let $\mathscr{A}_{n}$ be the Coxeter arrangement (known as the braid arrangement) of type $A_{n-1}$ in $\mathbb{R}^{n}$, consisting of the hyperplanes $x_{i}=x_{j}$ for $1 \leq i<j \leq n$. We have

$$
1+q \sum_{n \geq 1} \bar{\chi}_{\mathscr{A}_{n}}(q, t) \frac{x^{n}}{n !}=\left(\sum_{n \geq 0} t\left(\begin{array}{c}
n \\
2
\end{array}\right) \frac{x^{n}}{n !}\right)^{q} .
$$

Proof. For $n \geq 1$ we have $q \bar{\chi}_{\mathscr{A}_{n}}(q, t)=\sum_{p \in \mathbb{F}_{q}^{n}} t^{h(p)}$ for all powers of a large enough prime $q$, according to Theorem 3.3. For each $p \in \mathbb{F}_{q}^{n}$, if we let $A_{k}=\{i \in$ $\left.[n] \mid p_{i}=k\right\}$ for $0 \leq k \leq q-1$, then $h(p)=\left(\begin{array}{c}\left|A_{0}\right| \\ 2\end{array}\right)+\cdots+\left(\begin{array}{c}\left|A_{q-1}\right| \\ 2\end{array}\right)$. Thus

$$
q \bar{\chi}_{\mathscr{A}_{n}}(q, t)=\sum_{A_{0} \cup \cdots \cup A_{q-1}=[n]} t^{\left(\begin{array}{c}
\left|A_{0}\right| \\
2
\end{array}\right)+\cdots+\left(\begin{array}{c}
\left|A_{q-1}\right| \\
2
\end{array}\right)}
$$

summing over all weak ordered $q$-partitions of $[n]$; that is, ordered lists of $q$ pairwise disjoint, possibly empty sets whose union is $[n]$. The compositional formula for exponential generating functions [Bergeron et al. 1998; Stanley 1999, Proposition 5.1.3] implies the desired result. 
Theorem 4.2. Let $\mathscr{B}_{n}$ be the Coxeter arrangement of type $B_{n}$ in $\mathbb{R}^{n}$, consisting of the hyperplanes $x_{i}=x_{j}$ and $x_{i}+x_{j}=0$ for $1 \leq i<j \leq n$, and the hyperplanes $x_{i}=0$ for $1 \leq i \leq n$. We have

$$
\sum_{n \geq 0} \bar{\chi}_{\Re_{n}}(q, t) \frac{x^{n}}{n !}=\left(\sum_{n \geq 0} 2^{n} t\left(\begin{array}{c}
n \\
2
\end{array}\right) \frac{x^{n}}{n !}\right)^{\frac{q-1}{2}}\left(\sum_{n \geq 0} t^{n^{2}} \frac{x^{n}}{n !}\right) .
$$

Proof. Let $q$ be a power of a large enough prime, and let $s=\frac{q-1}{2}$. Now for each $p \in \mathbb{F}_{q}^{n}$, if we let $B_{k}=\left\{i \in[n] \mid p_{i}=k\right.$ or $\left.p_{i}=q-k\right\}$ for $0 \leq k \leq s$, we have that $h(p)=\left|B_{0}\right|^{2}+\left(\begin{array}{c}\left|B_{1}\right| \\ 2\end{array}\right)+\cdots+\left(\begin{array}{c}\left|B_{s}\right| \\ 2\end{array}\right)$. Also, given a weak ordered partition $\left(B_{0}, \ldots, B_{s}\right)$ of $[n]$, there are $2^{\left|B_{1}\right|+\cdots+\left|B_{s}\right|}$ points of $p$ which correspond to it: for each $i \in B_{k}$ with $k \neq 0$, we get to choose whether $p_{i}$ is equal to $k$ or to $q-k$. Therefore

$$
q \bar{\chi}_{\mathscr{B}_{n}}(q, t)=\sum_{B_{0} \cup \cdots \cup B_{s}=[n]} t^{\left|B_{0}\right|^{2}}\left(2^{\left|B_{1}\right|} t^{\mid\left(\begin{array}{c}
B_{1} \mid \\
2
\end{array}\right)}\right) \cdots\left(2^{\left|B_{s}\right|} t^{\left(\begin{array}{c}
\left|B_{s}\right| \\
2
\end{array}\right)}\right),
$$

and the compositional formula for exponential generating functions implies the theorem.

The proof of the next theorem is very similar to that of Theorem 4.2.

Theorem 4.3. Let $\mathscr{D}_{n}$ be the Coxeter arrangement of type $D_{n}$ in $\mathbb{R}^{n}$, consisting of the hyperplanes $x_{i}=x_{j}$ and $x_{i}+x_{j}=0$ for $1 \leq i<j \leq n$. We have

$$
\sum_{n \geq 0} \bar{\chi}_{\mathscr{D}_{n}}(q, t) \frac{x^{n}}{n !}=\left(\sum_{n \geq 0} 2^{n} t t^{\left(\begin{array}{c}
n \\
2
\end{array}\right)} \frac{x^{n}}{n !}\right)^{\frac{q-1}{2}}\left(\sum_{n \geq 0} t^{n(n-1)} \frac{x^{n}}{n !}\right) .
$$

Setting $t=0$ in Theorems 4.1, 4.2 and 4.3, it is easy to recover the formulas for the characteristic polynomials of the above arrangements:

$$
\begin{aligned}
& \chi_{\mathscr{A}_{n}}(q)=q(q-1)(q-2) \cdots(q-n+1), \\
& \chi_{\mathscr{B}_{n}}(q)=(q-1)(q-3) \cdots(q-2 n+1), \\
& \chi_{\mathscr{I}_{n}}(q)=(q-1)(q-3) \cdots(q-2 n+3)(q-n+1),
\end{aligned}
$$

which are well known; see for example [Stanley 2004].

\section{Two more examples.}

Theorem 4.4. Let $\mathscr{A}_{n}^{\#}$ be a generic deformation of the arrangement $\mathscr{A}_{n}$, consisting of the hyperplanes $x_{i}-x_{j}=a_{i j}(1 \leq i<j \leq n)$, where the $a_{i j}$ are generic real 
numbers ${ }^{1}$. For $n \geq 1$,

$$
q \bar{\chi}_{\mathscr{A}_{n}^{\#}}(q, t)=\sum_{\substack{F \text { forest } \\ \text { on }[n]}} q^{n-e(F)}(t-1)^{e(F)},
$$

where e $(F)$ denotes the number of edges of $F$. Also,

$$
1+q \sum_{n \geq 1} \bar{\chi}_{\mathbb{A}_{n}^{\#}}(q, t) \frac{x^{n}}{n !}=\exp \left(\frac{q}{t-1} \sum_{n \geq 0} n^{n-2} \frac{x^{n}(t-1)^{n}}{n !}\right) .
$$

Proof. It is possible to prove Theorem 4.4 using our finite field method, as we did in the previous section. However, it will be easier to proceed directly from (3-2), the definition of the coboundary polynomial.

To each subarrangement $\mathscr{B}$ of $\mathscr{A}_{n}^{\#}$ we can assign a graph $G_{\mathscr{B}}$ on the vertex set [n], by letting edge $i j$ be in $G_{\overparen{乃}}$ if and only if the hyperplane $x_{i}-x_{j}=a_{i j}$ is in $\mathscr{B}$. Since the $a_{i j} \mathrm{~s}$ are generic, the subarrangement $\mathscr{B}$ is central if and only if the corresponding graph $G_{\mathscr{B}}$ is a forest. For such a $\mathscr{B}$, it is clear that $|\mathscr{B}|=r(\mathscr{B})=$ $e\left(G_{\mathscr{\beta}}\right)$. Hence,

$$
\bar{\chi}_{\mathscr{A}_{n}^{\#}}(q, t)=\sum_{\substack{\mathscr{B} \subseteq \mathcal{A}_{n}^{\#} \\
\text { central }}} q^{r-r(\mathscr{B})}(t-1)^{|\mathscr{B}|}=\sum_{\begin{array}{c}
F \text { forest } \\
\text { on }[n]
\end{array}} q^{(n-1)-e(F)}(t-1)^{e(F)},
$$

proving the first claim. Now let $c(F)=n-e(F)$ be the number of connected components of $F$. We have

$$
\begin{aligned}
1+q \sum_{n \geq 1} \bar{\chi}_{\mathbb{A}_{n}^{\#}}(q, t) \frac{x^{n}}{n !} & =\sum_{n \geq 0} \sum_{\substack{F \text { forest } \\
\text { on }[n]}}\left(\frac{q}{t-1}\right)^{c(F)} \frac{x^{n}(t-1)^{n}}{n !} \\
& =\exp \left(\frac{q}{t-1} \sum_{n \geq 0} n^{n-2} \frac{x^{n}(t-1)^{n}}{n !}\right),
\end{aligned}
$$

using the exponential formula for exponential generating functions, and the fact that there are $n^{n-2}$ trees on $n$ labeled vertices [Stanley 1999].

Theorem 4.5. The threshold arrangement $\mathscr{T}_{n}$ in $\mathbb{R}^{n}$ consists of the hyperplanes $x_{i}+x_{j}=0$, for $1 \leq i<j \leq n$. For all $n \geq 0$ we have

$$
\bar{\chi}_{\mathscr{T}_{n}}(q, t)=\sum_{\substack{G \text { graph } \\ \text { on }[n]}} q^{b c(G)}(t-1)^{e(G)},
$$

\footnotetext{
${ }^{1}$ The $a_{i j}$ are "generic" if no $n$ of the hyperplanes have a nonempty intersection, and any nonempty intersection of $k$ hyperplanes has rank $k$. This can be achieved, for example, by requiring that the $a_{i j} \mathrm{~s}$ are linearly independent over the rational numbers. Almost all choices of the $a_{i j} \mathrm{~s}$ are generic.
} 
where $b c(G)$ is the number of connected components of $G$ which are bipartite, and $e(G)$ is the number of edges of $G$. Also,

$$
\sum_{n \geq 0} \bar{\chi}_{\mathscr{T}_{n}}(q, t) \frac{x^{n}}{n !}=\left(\sum_{n \geq 0} \sum_{k=0}^{n}\left(\begin{array}{l}
n \\
k
\end{array}\right) t^{k(n-k)} \frac{x^{n}}{n !}\right)^{\frac{q-1}{2}}\left(\sum_{n \geq 0} t t^{\left(\begin{array}{c}
n \\
2
\end{array}\right)} \frac{x^{n}}{n !}\right) .
$$

Proof. Once again, the proof of the first claim is easier using the definition of the coboundary polynomial. Every subarrangement $\mathscr{B}$ of $\mathscr{T}_{n}$ is central, and we can assign to it a graph $G_{\mathscr{乃}}$ as in the proof of Theorem 4.4. In view of (3-2), we only need to check that $r(\mathscr{B})=n-b c\left(G_{\mathscr{B}}\right)$ and $|\mathscr{乃}|=e\left(G_{\Re}\right)$. The second claim is trivial. To prove the first one, we show that $\operatorname{dim}(\cap \Re)=b c\left(G_{\Re}\right)$.

Consider a point $p$ in $\cap \Re$. We know that, if $a b$ is an edge in $G_{\mathscr{B}}$, then $p_{a}=-p_{b}$. If vertex $i$ is in a connected component $C$ of $G_{\mathscr{乃}}$, then the value of $p_{i}$ determines the value of $p_{j}$ for all $j$ in $C: p_{j}=p_{i}$ if there is a path of even length between $i$ and $j$, and $p_{j}=-p_{i}$ if there is a path of odd length between $i$ and $j$. If $C$ is bipartite, this determines the values of the $p_{j}$ s consistently. If $C$ is not bipartite, take a cycle of odd length and a vertex $k$ in it. We get that $p_{k}=-p_{k}$, so $p_{k}=0$; therefore we must have $p_{j}=0$ for all $j \in C$.

Therefore, to specify a point $p$ in $\bigcap \mathscr{B}$, we split $G_{\mathscr{B}}$ into its connected components. We know that $p_{i}=0$ for all $i$ in connected components which are not bipartite. To determine the remaining coordinates of $p$ we have to specify the value of $p_{j}$ for exactly one $j$ in each bipartite connected component. Therefore $\operatorname{dim}(\bigcap \mathscr{P})=b c\left(G_{\mathscr{\beta}}\right)$, as desired.

From this point, it is possible to prove the second claim of Theorem 4.5 using the compositional formula for exponential generating functions, in the same way that we proved Theorem 4.4. However, the work involved is considerable, and it is much simpler to use our finite field method, Theorem 3.3, in this case. The proof that we obtain is very similar to the proofs of Theorems 4.1, 4.2 and 4.3, so we omit the details.

\section{Deformations of the braid arrangement}

This section concerns the deformations of the braid arrangement of the form

$$
x_{i}-x_{j}=a_{1}, \ldots, a_{k} \quad 1 \leq i<j \leq n,
$$

where $A=\left\{a_{1}, \ldots, a_{k}\right\}$ is a fixed set of integers. Such arrangements have been studied extensively by Athanasiadis [2000] and Postnikov and Stanley [2000]. In this section we study the problem of finding their coboundary polynomials.

We proceed as follows. In the next subsection we introduce the family of graded $A$-graphs, and show in Proposition 5.6 that enumerating them is equivalent to the 
problem at hand. By understanding the structure of those graphs, we obtain Theorem 5.7, a formula for the generating function of our coboundary polynomials.

The formula provided by Theorem 5.7 is not very explicit, as one might expect from the fact that it applies to such a large family of arrangements. However, we show that for $A \subseteq\{-1,0,1\}, A$-graphs possess additional structure, which makes it possible to obtain very explicit answers. This is done starting on page 19 for the Linial, Shi, semiorder, and Catalan arrangements. As a consequence, we also obtain new formulas for the generating functions of alternating trees, labelled trees, semiorders, and Dyck paths.

\section{Enumerating graphs to compute coboundary polynomials.}

Definition 5.1. An exponential sequence of arrangements $\mathscr{E}=\left(\mathscr{E}_{0}, \mathscr{E}_{1}, \ldots\right)$ is a sequence of arrangements satisfying the following properties:

(1) $\mathscr{E}_{n}$ is an arrangement in $\mathbb{k}^{n}$, for some fixed field $\mathbb{k}$.

(2) Every hyperplane in $\mathscr{E}_{n}$ is parallel to some hyperplane in the braid arrangement $A_{n}$.

(3) For any subset $S$ of [n], the subarrangement $\mathscr{E}_{n} \subseteq \mathscr{E}_{n}$, which consists of the hyperplanes in $\mathscr{E}_{n}$ of the form $x_{i}-x_{j}=c$ with $i, j \in S$, is isomorphic to the arrangement $\mathscr{E}_{|S|}$.

The special case $t=0$ of the next result is due to Stanley [1996, Theorem 1.2]; we omit the proof, which is an easy extension of his.

Theorem 5.2. Let $\mathscr{E}=\left(\mathscr{E}_{0}, \mathscr{E}_{1}, \ldots\right)$ be an exponential sequence of arrangements. Then

$$
1+q \sum_{n \geq 1} \bar{\chi}_{\mathscr{E}_{n}}(q, t) \frac{x^{n}}{n !}=\left(\sum_{n \geq 0} \bar{\chi}_{\mathscr{E}_{n}}(1, t) \frac{x^{n}}{n !}\right)^{q} .
$$

The most natural examples of exponential sequences of arrangements are the following. Fix a set $A$ of $k$ distinct integers $a_{1}<\ldots<a_{k}$. Let $\mathscr{E}_{n}$ be the arrangement in $\mathbb{R}^{n}$ consisting of the hyperplanes

$$
x_{i}-x_{j}=a_{1}, \ldots, a_{k} \quad 1 \leq i<j \leq n .
$$

Then $\left(\mathscr{E}_{0}, \mathscr{E}_{1}, \ldots\right)$ is an exponential sequence of arrangements and Theorem 5.2 applies to this case. In fact, we can say much more about this type of arrangement.

After proving the results in this section, we found out that Postnikov and Stanley [2000] had used similar techniques in computing the characteristic polynomials of these types of arrangements. Therefore, for consistency, we will use the terminology that they introduced. 
Definition 5.3. A graded graph is a triple $G=\left(V_{G}, E_{G}, h_{G}\right)$, where $V_{G}$ is a linearly ordered set of vertices (usually $V_{G}=[n]$ ), $E_{G}$ is a set of undirected edges, and $h_{G}$ is a function $h_{G}: V \rightarrow \mathbb{N}$, called a grading.

We will drop the subscripts when the underlying graded graph is clear. We will refer to $h(v)$ as the height of vertex $v$. The height of $G$, denoted $h(G)$, is the largest height of a vertex of $G$.

Definition 5.4. Let $G$ be a graded graph and $r$ be a nonnegative integer. Let the $r$ th level of $G$ be the set of vertices $v$ such that $h(v)=r$. Say $G$ is planted if each one of its connected components has a vertex on the 0th level.

Definition 5.5. If $u<v$ are connected by edge $e$ in a graded graph $G$, let the type of $e$ be $s(e)=h(u)-h(v)$. Say $G$ is an A-graph if the types of all edges of $G$ are in $A=\left\{a_{1}, \ldots, a_{k}\right\}$.

Recall that, for a graph $G$, we let $e(G)$ be the number of edges and $c(G)$ be the number of connected components of $G$.

Proposition 5.6. Let $A=\left\{a_{1}, \ldots, a_{k}\right\}$, and let $\mathscr{E}_{n}$ be the arrangement

$$
x_{i}-x_{j}=a_{1}, \ldots, a_{k} \quad 1 \leq i<j \leq n .
$$

Then, for $n \geq 1$,

$$
q \bar{\chi}_{\mathscr{E}_{n}}(q, t)=\sum_{G} q^{c(G)}(t-1)^{e(G)},
$$

where the sum is over all planted graded A-graphs on $[n]$.

Proof. We associate to each planted graded $A$-graph $G=(V, E, h)$ on $[n]$ a central subarrangement $\mathscr{A}_{G}$ of $\mathscr{E}_{n}$. It consists of the hyperplanes $x_{i}-x_{j}=h(i)-h(j)$, for each $i<j$ such that $i j$ is an edge in $G$. This is a subarrangement of $\mathscr{E}_{n}$ because $h(i)-h(j)$, the type of edge $i j$, is in $A$. It is central because the point $(h(1), \ldots, h(n)) \in \mathbb{R}^{n}$ belongs to all these hyperplanes.

Example. Consider an arrangement $\mathscr{E}_{8}$ in $\mathbb{R}^{8}$, with a subarrangement consisting of the hyperplanes $x_{1}-x_{2}=4, x_{1}-x_{3}=-1, x_{1}-x_{6}=0, x_{1}-x_{8}=1, x_{2}-x_{3}=-5$ and $x_{4}-x_{7}=2$. Figure 1 shows the planted graded $A$-graph corresponding to this subarrangement.

This is in fact a bijection between planted graded $A$-graphs on $[n]$ and central subarrangements of $\mathscr{E}_{n}$. To see this, take a central subarrangement $\mathscr{A}$. We will recover the planted graded $A$-graph $G$ that it came from. For each pair $(i, j)$ with $1 \leq i<j \leq n, \mathscr{A}$ can have at most one hyperplane of the form $x_{i}-x_{j}=a_{t}$. If this hyperplane is in $\mathscr{A}$, we must put edge $i j$ in $G$, and demand that the heights $h(i)$ and $h(j)$ satisfy $h(i)-h(j)=a_{t}$. When we do this for all the hyperplanes in $\mathscr{A}$, the height requirements that we introduce are consistent, because $\mathscr{A}$ is central. 


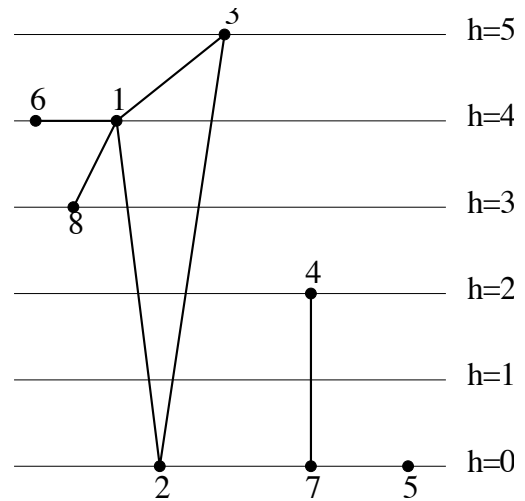

Figure 1. The planted graded $A$-graph for the subarrangement of $\mathscr{E}_{8}$ in the example from the proof of Proposition 5.6.

However, these requirements do not fully determine the heights of the vertices; they only determine the relative heights within each connected component of $G$. Since we want $G$ to be planted, we demand that the vertices with the lowest height in each connected component of $G$ should have height 0 . This does determine $G$ completely, and clearly $\mathscr{A}=\mathscr{A}_{G}$.

With this bijection in hand, and keeping (3-2) in mind, it remains to show that $r\left(\mathscr{A}_{G}\right)=n-c(G)$ and $\left|\mathscr{A}_{G}\right|=e(G)$. The second of these claims is trivial. We omit the proof of the first one which is very similar to, and simpler than, that of $r(\mathscr{B})=n-b c\left(G_{\mathscr{B}}\right)$ in our proof of Theorem 4.5.

Theorem 5.7. Let $A=\left\{a_{1}, \ldots, a_{n}\right\}$ and let $\mathscr{E}_{n}$ be the arrangement

$$
x_{i}-x_{j}=a_{1}, \ldots, a_{k} \quad 1 \leq i<j \leq n .
$$

Let

$$
A_{r}(t, x)=\sum_{n \geq 0}\left(\sum_{f:[n] \rightarrow[r]} t^{a(f)}\right) \frac{x^{n}}{n !},
$$

where the inside sum is over all functions $f:[n] \rightarrow[r]$, and

$$
a(f)=\#\{(i, j) \mid 1 \leq i<j \leq n, f(i)-f(j) \in A\} .
$$

Then

$$
1+q \sum_{n \geq 1} \bar{\chi}_{\mathscr{E}_{n}}(q, t) \frac{x^{n}}{n !}=\left(\lim _{r \rightarrow \infty} \frac{A_{r}(t, x)}{A_{r-1}(t, x)}\right)^{q} .
$$

Remark. The limit in (5-4) is a limit in the sense of convergence of formal power series. Let $F_{1}(t, x), F_{2}(t, x), \ldots$ be a sequence of formal power series. We say that $\lim _{n \rightarrow \infty} F_{n}(t, x)=F(t, x)$ if for all $a$ and $b$ there exists a constant $N(a, b)$ such that, for all $n$ larger than $N(a, b)$, the coefficient of $t^{a} x^{b}$ in $F_{n}(t, x)$ is equal 
to the coefficient of $t^{a} x^{b}$ in $F(t, x)$. For more on this notion of convergence, see [Niven 1969] or [Stanley 1997, Section 1.1].

Proof of Theorem 5.7. Let $v(G)$ be the number of vertices of graph $G$. First we prove that

$$
A_{r}(t, x)=\sum_{G}(t-1)^{e(G)} \frac{x^{v(G)}}{v(G) !}
$$

where the sum is over all graded $A$-graphs $G$ on $[n]$ of height less than $r$. The coefficient of $\frac{x^{n}}{n !}$ in the right-hand side of (5-5) is $\sum_{G}(t-1)^{e(G)}$, summing over all graded $A$-graphs $G$ on $[n]$ with height less than $r$. We have

$$
\begin{aligned}
\sum_{G}(t-1)^{e(G)} & =\sum_{h:[n] \rightarrow[0, r-1]} \sum_{G: h_{G}=h}(t-1)^{e(G)} \\
& =\sum_{h:[n] \rightarrow[0, r-1]}(1+(t-1))^{a(h)}=\sum_{f:[n] \rightarrow[r]} t^{a(f)} .
\end{aligned}
$$

The only tricky step here is the second: if we want all graded $A$-graphs $G$ on $[n]$ with a specified grading $h$, we need to consider the possible choices of edges of the graph. Any edge $i j$ can belong to the graph, as long as $h(i)-h(j) \in A$, so there are $a(h)$ possible edges.

Equation (5-5) suggests the following definitions. Let

$$
B_{r}(t, x)=\sum_{G} t^{e(G)} \frac{x^{v(G)}}{v(G) !}
$$

where the sum is over all planted graded $A$-graphs $G$ of height less than $r$, and let

$$
B(t, x)=\sum_{G} t^{e(G)} \frac{x^{v(G)}}{v(G) !}
$$

where the sum is over all planted graded $A$-graphs $G$.

The equation

$$
1+q \sum_{n \geq 1} \bar{\chi}_{\mathscr{E}_{n}}(q, t) \frac{x^{n}}{n !}=B(t-1, x)^{q},
$$

follows from Proposition 5.6, using either Theorem 5.2 or the compositional formula for exponential generating functions.

Now we claim that $B(t, x)=\lim _{r \rightarrow \infty} B_{r}(t, x)$. In a planted graded $A$-graph $G$ with $e$ edges and $v$ vertices, each vertex has a path of length at most $v$ that connects it to a vertex on the 0 th level. Therefore $h(G) \leq v \cdot \max \left(\left|a_{1}\right|, \ldots,\left|a_{k}\right|\right)$, so the coefficients of $t^{e} \frac{x^{v}}{v !}$ in $B_{r}(t, x)$ and $B(t, x)$ are equal for $r>v \cdot \max \left(\left|a_{1}\right|, \ldots,\left|a_{k}\right|\right)$. 
Then it is not difficult to show that

$$
B(t-1, x)=\lim _{r \rightarrow \infty} B_{r}(t-1, x) .
$$

Here it is necessary to check that $B(t-1, x)$ is, indeed, a formal power series. This follows from the observation that the coefficient of $\frac{x^{n}}{n !}$ in $B(t, x)$ is a polynomial in $t$ of degree at most $\left(\begin{array}{l}n \\ 2\end{array}\right)$. Once again, see [Stanley 1997, Section 1.1] for more information on these technical details.

Next, we show that

$$
B_{r}(t-1, x)=A_{r}(t, x) / A_{r-1}(t, x)
$$

or, equivalently, that $A_{r}(t, x)=B_{r}(t-1, x) A_{r-1}(t, x)$. The multiplication formula for exponential generating functions [Stanley 1999, Proposition 5.1.1] and (5-5) give us a combinatorial interpretation of this identity. We need to show that the ways of putting the structure of a graded $A$-graph $G$ with $h(G)<r$ on $[n]$ can be put in correspondence with the ways of doing the following: first splitting $[n]$ into two disjoint sets $S_{1}$ and $S_{2}$, then putting the structure of a planted graded $A$-graph $G_{1}$ with $h\left(G_{1}\right)<r$ on $S_{1}$, and then putting the structure of a graded $A$ graph $G_{2}$ with $h\left(G_{2}\right)<r-1$ on $S_{2}$. We also need that, in that correspondence, $(t-1)^{e(G)}=(t-1)^{e\left(G_{1}\right)}(t-1)^{e\left(G_{2}\right)}$.

We do this as follows. Let $G$ be a graded $A$-graph $G$ with $h(G)<r$. Let $G_{1}$ be the union of the connected components of $G$ which contain a vertex on the 0th level. Put a grading on $G_{1}$ by defining $h_{G_{1}}(v)=h_{G}(v)$ for $v \in G_{1}$. Let $G_{2}=G-G_{1}$. It is clear that $h_{G}(v) \geq 1$ for all $v \in G_{2}$; therefore we can put a grading on $G_{2}$ by defining $h_{G_{2}}(v)=h_{G}(v)-1$ for $v \in G_{2}$. Then $G_{1}$ is a planted
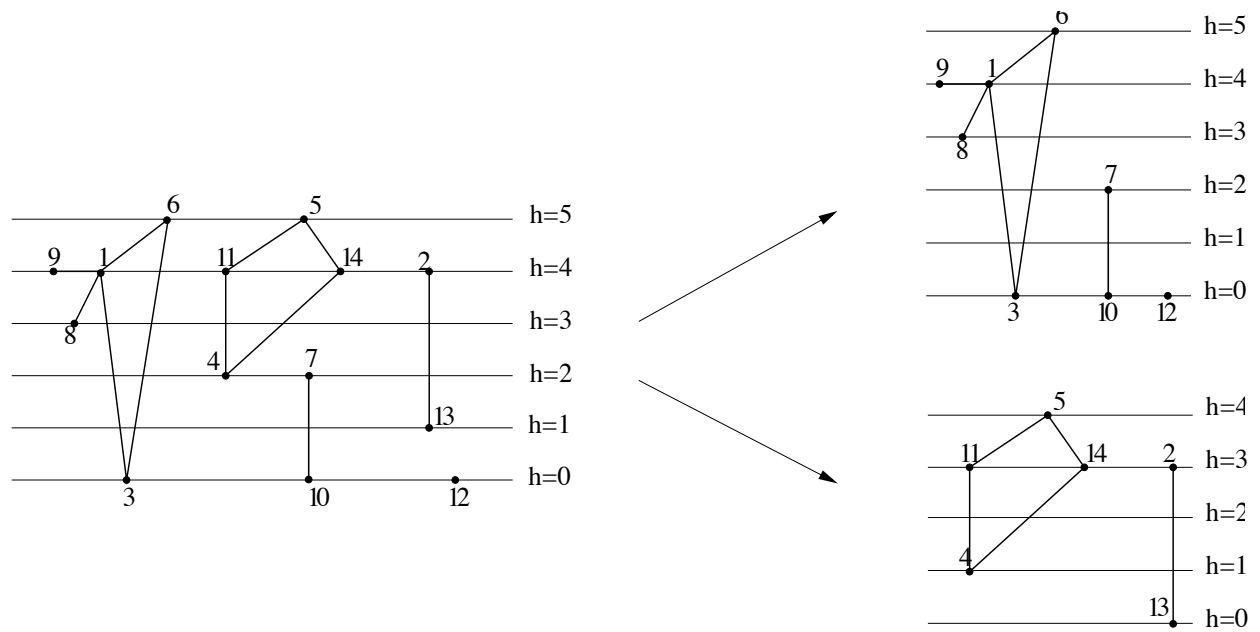

Figure 2. The decomposition of a graded $A$-graph. 
graded $A$-graph with $h\left(G_{1}\right)<r, G_{2}$ is a graded $A$-graph with $h\left(G_{2}\right)<r-1$, and $(t-1)^{e(G)}=(t-1)^{e\left(G_{1}\right)}(t-1)^{e\left(G_{2}\right)}$. It is clear how to recover $G$ from $G_{1}$ and $G_{2}$. Figure 2 illustrates this bijection with an example.

Now we just have to put together (5-6), (5-7) and (5-8) to complete the proof of Theorem 5.7.

Subarrangements of the Catalan arrangement. The Catalan arrangement $C_{n}$ in $\mathbb{R}^{n}$ consists of the hyperplanes

$$
x_{i}-x_{j}=-1,0,1 \quad 1 \leq i<j \leq n .
$$

When the arrangement in Theorem 5.7 is a subarrangement of the Catalan arrangement, we can say more about the power series $A_{r}$ of (5-3). Let

$$
A(t, x, y)=\sum_{r} A_{r}(t, x) y^{r}=\sum_{n \geq 0} \sum_{r \geq 0}\left(\sum_{f:[n] \rightarrow[r]} t^{a(f)}\right) \frac{x^{n}}{n !} y^{r}
$$

and let

$$
S(t, x, y)=\sum_{n \geq 0} \sum_{r \geq 0}\left(\sum_{f:[n] \rightarrow[r]} t^{a(f)}\right) \frac{x^{n}}{n !} y^{r}
$$

where the inner sum is over all surjective functions $f:[n] \rightarrow[r]$. The following proposition reduces the computation of $A(t, x, y)$ to the computation of $S(t, x, y)$, which is often easier in practice.

Proposition 5.8. If $A \subseteq\{-1,0,1\}$ in the notation of Theorem 5.7, we have

$$
A(t, x, y)=\frac{S(t, x, y)}{1-y S(t, x, y)}
$$

Proof. Once again, we think of this as an identity about exponential generating functions in the variable $x$. Fix $n, r$, and a function $f:[n] \rightarrow[r]$. Write $[r]-$ $\operatorname{Im} f=\left\{m_{1}, m_{1}+m_{2}, \ldots, m_{1}+\cdots+m_{k-1}\right\}$, so the image of $f$ is $M_{1} \cup \cdots \cup$ $M_{k}=\left\{1, \ldots, m_{1}-1\right\} \cup\left\{m_{1}+1, \ldots, m_{1}+m_{2}-1\right\} \cup \cdots \cup\left\{m_{1}+\cdots+m_{k-1}+\right.$ $\left.1, \ldots, m_{1}+\cdots+m_{k}-1\right\}$. Here $m_{1}, \ldots, m_{k}$ are arbitrary positive integers such that $m_{1}+\cdots+m_{k}-1=r$. For $1 \leq i \leq k$, let $f_{i}$ be the restriction of $f$ to $f^{-1}\left(M_{i}\right)$; it maps $f^{-1}\left(M_{i}\right)$ surjectively to $M_{i}$. Then we can "decompose" $f$ in a unique way into the $k$ surjective functions $f_{1}, \ldots, f_{k}$. The weight $w(f)$ corresponding to $f$ in $A(t, x, y)$ is $t^{a(f)} y^{r}$, while the weight $w\left(f_{i}\right)$ corresponding to $f_{i}$ in $S(t, x, y)$ is $t^{a\left(f_{i}\right)} y^{m_{i}-1}$.

Now observe that $a(f)=a\left(f_{1}\right)+\cdots+a\left(f_{k}\right)$ : whenever we have a pair of numbers $1 \leq i<j \leq n$ counted by $a(f)$, since $f(i)-f(j) \in\{-1,0,1\}$, we know that $f(i)$ and $f(j)$ must be in the same $M_{h}$. Therefore $i$ and $j$ are in the same $f^{-1}\left(M_{h}\right)$, and this pair is also counted in $a\left(f_{h}\right)$. We also have that $r=$ 
$\left(m_{1}-1\right)+\cdots+\left(m_{k}-1\right)+(k-1)$. Therefore $w(f)=w\left(f_{1}\right) \cdots w\left(f_{k}\right) y^{k-1}$. It follows from the compositional formula for exponential generating functions that

$$
A(t, x, y)=\sum_{k \geq 1} S(t, x, y)^{k} y^{k-1}=\frac{S(t, x, y)}{1-y S(t, x, y)},
$$

as desired.

Considering the different subsets of $\{-1,0,1\}$, we get six nonisomorphic subarrangements of the Catalan arrangement. They come from the subsets $\varnothing,\{0\},\{1\}$, $\{0,1\},\{-1,1\}$ and $\{-1,0,1\}$. The corresponding subarrangements are the empty arrangement, the braid arrangement, the Linial arrangement, the Shi arrangement, the semiorder arrangement, and the Catalan arrangement, respectively. The empty arrangement is trivial, and the braid arrangement was already treated in detail starting on page 10. We now have a technique that lets us talk about the remaining four arrangements under the same framework. We will do this in the remainder of this section.

The Linial arrangement. The Linial arrangement $\mathscr{L}_{n}$ consists of the hyperplanes $x_{i}-x_{j}=1$ for $1 \leq i<j \leq n$. This arrangement was first considered by Linial and Ravid. It was later studied by Athanasiadis [1996] and Postnikov and Stanley [2000], who independently computed the characteristic polynomial of $\mathscr{L}_{n}$ :

$$
\chi_{\mathscr{L}_{n}}(q)=\frac{q}{2^{n}} \sum_{k=0}^{n}\left(\begin{array}{l}
n \\
k
\end{array}\right)(q-k)^{n-1} .
$$

They also put the regions of $\mathscr{L}_{n}$ in bijection with several different sets of combinatorial objects. Perhaps the simplest such set is the set of alternating trees on $[n+1]$ : the trees such that every vertex is either larger or smaller than all its neighbors.

Now we present the consequences of Proposition 5.8 and Propositions 5.6 and 5.7 for the Linial arrangement. Say that a poset $P$ on $[n]$ is naturally labeled if $i<j$ in $P$ implies $i<j$ in $\mathbb{Z}^{+}$.

Proposition 5.9. For all $n \geq 1$ we have

$$
q \bar{\chi}_{\mathscr{L}_{n}}(q, t)=\sum_{P} q^{c(P)}(t-1)^{e(P)}
$$

where the sum is over all naturally labeled, graded posets $P$ on $[n]$. Here $c(P)$ and $e(P)$ denote the number of components and edges of the Hasse diagram of $P$, respectively.

Proof. There is an obvious bijection between Hasse diagrams of naturally labeled graded posets on $[n]$ and planted graded $\{1\}$-graphs on $[n]$. The result then follows immediately from Proposition 5.6. 
Theorem 5.10. Let

$$
A_{r}(t, x)=\sum_{n \geq 0}\left(\sum_{f:[n] \rightarrow[r]} t^{i d(f)}\right) \frac{x^{n}}{n !}
$$

where id $(f)$ denotes the number of inverse descents of the word $f(1) \ldots f(n)$, that $i$, the number of pairs $(i, j)$ with $1 \leq i<j \leq n$ such that $f(i)-f(j)=1$. Then

$$
1+q \sum_{n \geq 1} \bar{\chi}_{\mathscr{L}_{n}}(q, t) \frac{x^{n}}{n !}=\left(\lim _{r \rightarrow \infty} \frac{A_{r}(t, x)}{A_{r-1}(t, x)}\right)^{q} .
$$

Proof. This is immediate from Theorem 5.7.

Recall that the descents of a permutation $\sigma=\sigma_{1} \ldots \sigma_{r} \in S_{r}$ are the indices $i$ such that $\sigma_{i}>\sigma_{i+1}$. For more information about descents, see [Stanley 1997, Section 1.3], for example. We call $i d(f)$ the number of inverse descents, because they generalize descents in the following way. If $\pi:[r] \rightarrow[r]$ is a permutation, then $i d(\pi)$ is the number of descents of the permutation $\pi^{-1}$.

We can use Theorem 5.10 to say more about the characteristic polynomial of $\mathscr{L}_{n}$ which, as discussed on page 4 , is given by $\chi_{\mathscr{L}_{n}}(q)=q \bar{\chi}_{\mathscr{L}_{n}}(q, 0)$.

Theorem 5.11. Let

$$
\frac{1+y e^{x(1+y)}}{1-y^{2} e^{x(1+y)}}=\sum_{r \geq 0} A_{r}(x) y^{r}
$$

Then we have

$$
\sum_{n \geq 0} \chi_{\mathscr{L}_{n}}(q) \frac{x^{n}}{n !}=\left(\lim _{r \rightarrow \infty} \frac{A_{r}(x)}{A_{r-1}(x)}\right)^{q} .
$$

In particular, if $f_{n}$ is the number of alternating trees on $[n+1]$, we have

$$
\sum_{n \geq 0}(-1)^{n} f_{n} \frac{x^{n}}{n !}=\lim _{r \rightarrow \infty} \frac{A_{r-1}(x)}{A_{r}(x)} .
$$

Proof. In view of Theorem 5.10 and Proposition 5.8, we compute $S(0, x, y)$. From (5-9), the coefficient of $\frac{x^{n}}{n !} y^{r}$ in $S(0, x, y)$ is equal to the number of surjective functions $f:[n] \rightarrow[r]$ with no inverse descents. These are just the nondecreasing surjective functions $f:[n] \rightarrow[r]$. For $n \geq 1$ and $r \geq 1$ there are $\left(\begin{array}{l}n-1 \\ r-1\end{array}\right)$ such functions, and for $n=r=0$ there is one such function. In the other cases there are none. Therefore

$$
S(0, x, y)=1+\sum_{n \geq 1} \sum_{r \geq 1}\left(\begin{array}{l}
n-1 \\
r-1
\end{array}\right) \frac{x^{n}}{n !} y^{r}=1+\sum_{n \geq 1} \frac{x^{n}}{n !} y(1+y)^{n-1}=\frac{1+y e^{x(1+y)}}{1+y} .
$$


Proposition 5.8 then implies that

$$
A(0, x, y)=\frac{1+y e^{x(1+y)}}{1-y^{2} e^{x(1+y)}},
$$

in agreement with (5-10), and the theorem follows.

The Shi arrangement. The Shi arrangement $\mathscr{Y}_{n}$ consists of the hyperplanes $x_{i}-$ $x_{j}=0,1$ for $1 \leq i<j \leq n$. Shi [1986, Chapter 7; 1987] first considered this arrangement, and showed that first considered this arrangement, and showed that it has $(n+1)^{n-1}$ regions. Headley [1994, Chapter VI; 1997] later computed the characteristic polynomial of later computed the characteristic polynomial of $\mathscr{S}_{n}$ :

$$
\chi_{\varphi_{n}}(q)=q(q-n)^{n-1} .
$$

Stanley [1996; 1998b] gave a nice bijection between regions of the Shi arrangement and parking functions of length $n$. Parking functions were first introduced by Konheim and Weiss [1966]; for more information, see [Stanley 1999, Exercise 5.49].

For the Shi arrangement, we can say the following.

Theorem 5.12. Let

$$
A_{r}(x)=\sum_{n=0}^{r}(r-n)^{n} \frac{x^{n}}{n !}
$$

Then we have

$$
\sum_{n \geq 0} \chi \varphi_{n}(q) \frac{x^{n}}{n !}=\left(\lim _{r \rightarrow \infty} \frac{A_{r}(x)}{A_{r-1}(x)}\right)^{q} .
$$

In particular, we have

$$
\sum_{n \geq 0}(-1)^{n}(n+1)^{n-1} \frac{x^{n}}{n !}=\lim _{r \rightarrow \infty} \frac{A_{r-1}(x)}{A_{r}(x)} .
$$

Proof. We proceed in the same way that we did in Theorem 5.11. In this case, we need to compute the number of surjective functions $f:[n] \rightarrow[r]$ such that $f(i)-f(j)$ is never equal to 0 or 1 for $i<j$. These are just the surjective, strictly increasing functions. There is only one of them when $n=r$, and there are none when $n \neq r$. Hence

$$
S(0, x, y)=\sum_{n \geq 0} \frac{x^{n}}{n !} y^{n}=e^{x y} .
$$

The rest follows easily by computing $A(0, x, y)$ and $A_{r}(x)$ explicitly. 
The semiorder arrangement. The semiorder arrangement $\Phi_{n}$ consists of the hyperplanes $x_{i}-x_{j}=-1,1$ for $1 \leq i<j \leq n$. A semiorder on $[n]$ is a poset $P$ on $[n]$ for which there exist $n$ unit intervals $I_{1}, \ldots, I_{n}$ of $\mathbb{R}$, such that $i<j$ in $P$ if and only if $I_{i}$ is disjoint from $I_{j}$ and to the left of it. It is known [Scott and Suppes 1958] that a poset is a semiorder if and only if it does not contain a subposet isomorphic to $3+\mathbf{1}$ or $\mathbf{2}+\mathbf{2}$. We are interested in semiorders because the number of regions of $\Phi_{n}$ is equal to the number of semiorders on $[n]$, as shown in [Postnikov and Stanley 2000] and [Stanley 1996].

Theorem 5.13. Let

$$
\frac{1-y+y e^{x}}{1-y+y^{2}-y^{2} e^{x}}=\sum_{r \geq 0} A_{r}(x) y^{r} .
$$

Then we have

$$
\sum_{n \geq 0} \chi_{\Phi_{n}}(q) \frac{x^{n}}{n !}=\left(\lim _{r \rightarrow \infty} \frac{A_{r}(x)}{A_{r-1}(x)}\right)^{q} .
$$

In particular, if $i_{n}$ is the number of semiorders on $[n]$, we have

$$
\sum_{n \geq 0}(-1)^{n} i_{n} \frac{x^{n}}{n !}=\lim _{r \rightarrow \infty} \frac{A_{r-1}(x)}{A_{r}(x)} .
$$

Proof. In this case, $S(0, x, y)$ counts surjective functions $f:[n] \rightarrow[r]$ such that $f(i)-f(j)$ is never equal to 1 for $i \neq j$. Such a function has to be constant; so it can only exist (and is unique) if $n \geq 1$ and $r=1$ or if $n=r=0$. Thus

$$
S(0, x, y)=1+\left(e^{x}-1\right) y
$$

and the rest follows easily.

The Catalan arrangement. The Catalan arrangement $C_{n}$ consists of the hyperplanes $x_{i}-x_{j}=-1,0,1$ for $1 \leq i<j \leq n$. Stanley [1996] observed that the number of regions of this arrangement is $n ! C_{n}$, where $C_{n}=\frac{1}{n+1}\left(\begin{array}{c}2 n \\ n\end{array}\right)$ is the $n$ th Catalan number. For (much) more information on the Catalan numbers; see [Stanley 1999, Chapter 6], especially Exercise 6.19.

Theorem 5.14. Let

$$
A_{r}(x)=\sum_{n=0}^{\left\lfloor\frac{r+1}{2}\right\rfloor}\left(\begin{array}{c}
r-n+1 \\
n
\end{array}\right) x^{n} .
$$

Then we have

$$
\sum_{n \geq 0} \chi_{C_{n}}(q) \frac{x^{n}}{n !}=\left(\lim _{r \rightarrow \infty} \frac{A_{r}(x)}{A_{r-1}(x)}\right)^{q}
$$


In particular,

$$
\frac{\sqrt{1+4 x}-1}{2 x}=\sum_{n \geq 0}(-1)^{n} C_{n} x^{n}=\lim _{r \rightarrow \infty} \frac{A_{r-1}(x)}{A_{r}(x)} .
$$

Proof. If $f:[n] \rightarrow[r]$ is a surjective function such that $f(i)-f(j)$ is never equal to $-1,0$ or 1 for $i \neq j$, then $n=r=0$ or $n=r=1$. Thus $S(x, y, 0)=1+x y$, and the rest of the proof is straightforward.

The polynomial $A_{r}(x)$ is a simple transformation of the Fibonacci polynomial. The number of words of length $r$, consisting of $0 \mathrm{~s}$ and $1 \mathrm{~s}$, which do not contain two consecutive $1 \mathrm{~s}$, is equal to $F_{r+2}$, the $(r+2)$ th Fibonacci number. It is easy to see that the polynomial $A_{r}(x)$ counts those words according to the number of $1 \mathrm{~s}$ they contain. In particular, $A_{r}(1)=F_{r+2}$.

We close with an amusing observation. Irresponsibly ${ }^{2}$ plugging $x=1$ into (5-11), we obtain an unconventional "proof" of the asymptotic rate of growth of Fibonacci numbers:

$$
\frac{\sqrt{5}-1}{2}=\lim _{r \rightarrow \infty} \frac{F_{r-1}}{F_{r}}
$$

\section{Acknowledgments}

This work is Chapter 2 of my Ph.D. thesis [Ardila 2003]. I would like to thank my advisor, Richard Stanley, for introducing me to the topic of hyperplane arrangements, and for asking some of the questions which led to this investigation. I am also grateful to Ira Gessel and Vic Reiner for helpful discussions on this subject. Finally, I thank the referee for a careful reading of the manuscript, and valuable suggestions for improvement.

\section{References}

[Ardila 2003] F. Ardila, Enumerative and algebraic aspects of matroids and hyperplane arrangements, Ph.D. thesis, Massachusetts Institute of Technology, Cambridge, MA, 2003.

[Ardila 2004] F. Ardila, "Semimatroids and their Tutte polynomials", preprint, Massachusetts Institute of Technology, 2004. To appear in Revista Colombiana de Matemáticas. math.CO/0409003

[Athanasiadis 1996] C. A. Athanasiadis, "Characteristic polynomials of subspace arrangements and finite fields", Adv. Math. 122:2 (1996), 193-233. MR 97k:52012 Zbl 0872.52006

[Athanasiadis 2000] C. A. Athanasiadis, "Deformations of Coxeter hyperplane arrangements and their characteristic polynomials", pp. 1-26 in Arrangements (Tokyo, 1998), edited by M. Falk and H. Terao, Adv. Stud. Pure Math. 27, Kinokuniya, Tokyo, 2000. MR 2001i:52035 Zbl 0976.32016

\footnotetext{
${ }^{2}$ We are not necessarily justified in doing this, since we have only proved equality in (5-11) as formal power series!
} 
[Bergeron et al. 1998] F. Bergeron, G. Labelle, and P. Leroux, Combinatorial species and treelike structures, Encyclopedia of Mathematics and its Applications 67, Cambridge University Press, Cambridge, 1998. MR 2000a:05008 Zbl 0888.05001

[Björner and Brenti 2005] A. Björner and F. Brenti, Combinatorics of Coxeter groups, Graduate Texts in Mathematics 231, Springer, New York, 2005. MR 2006d:05001 Zbl 02186170

[Björner et al. 1993] A. Björner, M. Las Vergnas, B. Sturmfels, N. White, and G. M. Ziegler, Oriented matroids, Encyclopedia of Mathematics and its Applications 46, Cambridge University Press, Cambridge, 1993. MR 95e:52023 Zbl 0773.52001

[Brylawski and Oxley 1992] T. Brylawski and J. Oxley, "The Tutte polynomial and its applications", pp. 123-225 in Matroid applications, Encyclopedia of Mathematics and its Applications 40, Cambridge Univ. Press, Cambridge, 1992. MR 93k:05060 Zbl 0769.05026

[Crapo 1968] H. H. Crapo, "Möbius inversion in lattices", Arch. Math. (Basel) 19 (1968), 595-607. MR 39 \#6791 Zbl 0208.29303

[Crapo 1969] H. H. Crapo, “The Tutte polynomial”, Aequationes Math. 3 (1969), 211-229. MR 41 \#6705 Zbl 0197.50202

[Crapo and Rota 1970] H. H. Crapo and G.-C. Rota, On the foundations of combinatorial theory: Combinatorial geometries, preliminary ed., MIT Press, Cambridge, MA, 1970. MR 45 \#74 Zbl 0216.02101

[Greene 1976] C. Greene, "Weight enumeration and the geometry of linear codes", Studies in Appl. Math. 55:2 (1976), 119-128. MR 56 \#5335 Zbl 0331.05019

[Headley 1994] P. Headley, Reduced expressions in infinite Coxeter groups, Ph.D. thesis, University of Michigan, Ann Arbor, 1994.

[Headley 1997] P. Headley, "On a family of hyperplane arrangements related to the affine Weyl groups”, J. Algebraic Combin. 6:4 (1997), 331-338. MR 98e:52010 Zbl 0911.52009

[Humphreys 1990] J. E. Humphreys, Reflection groups and Coxeter groups, Cambridge Studies in Advanced Mathematics 29, Cambridge University Press, Cambridge, 1990. MR 92h:20002 Zbl 0768.20016

[Konheim and Weiss 1966] A. G. Konheim and B. Weiss, "An occupancy discipline and applications.”, SIAM J. Appl. Math. 14 (1966), 1266-1274. Zbl 0201.50204

[Niven 1969] I. Niven, "Formal power series", Amer. Math. Monthly 76 (1969), 871-889. MR 40 \#5606 Zbl 0184.29603

[Orlik and Solomon 1980] P. Orlik and L. Solomon, "Combinatorics and topology of complements of hyperplanes", Invent. Math. 56:2 (1980), 167-189. MR 81e:32015 Zbl 0432.14016

[Orlik and Terao 1992] P. Orlik and H. Terao, Arrangements of hyperplanes, Grundlehren der Math. Wiss. 300, Springer, Berlin, 1992. MR 94e:52014 Zbl 0757.55001

[Postnikov and Stanley 2000] A. Postnikov and R. P. Stanley, "Deformations of Coxeter hyperplane arrangements", J. Combin. Theory Ser. A 91 (2000), 544-597. MR 2002g:52032 Zbl 0962.05004

[Reiner 1999] V. Reiner, "An interpretation for the Tutte polynomial", European J. Combin. 20:2 (1999), 149-161. MR 2000b:05037 Zbl 0924.05015

[Scott and Suppes 1958] D. Scott and P. Suppes, "Foundational aspects of theories of measurement", J. Symb. Logic 23 (1958), 113-128. MR 22 \#6716 Zbl 0084.24603

[Shi 1986] J. Y. Shi, The Kazhdan-Lusztig cells in certain affine Weyl groups, Lecture Notes in Mathematics 1179, Springer, Berlin, 1986. MR 87i:20074 Zbl 0582.20030

[Shi 1987] J. Y. Shi, "Sign types corresponding to an affine Weyl group", J. London Math. Soc. (2) 35:1 (1987), 56-74. MR 88g:20103b Zbl 0681.20033 
[Stanley 1996] R. P. Stanley, "Hyperplane arrangements, interval orders, and trees", Proc. Nat. Acad. Sci. U.S.A. 93:6 (1996), 2620-2625. MR 97i:52013 Zbl 0848.05005

[Stanley 1997] R. P. Stanley, Enumerative combinatorics, vol. 1, corrected ed., Cambridge Studies in Advanced Mathematics 49, Cambridge University Press, Cambridge, 1997. MR 98a:05001 Zbl 0889.05001

[Stanley 1998a] R. P. Stanley, "Graph colorings and related symmetric functions: ideas and applications: a description of results, interesting applications, \& notable open problems", Discrete Math. 193:1-3 (1998), 267-286. MR 2000c:05152 Zbl 1061.05508

[Stanley 1998b] R. P. Stanley, "Hyperplane arrangements, parking functions and tree inversions", pp. 359-375 in Mathematical essays in honor of Gian-Carlo Rota (Cambridge, MA, 1996), edited by B. Sagan and R. Stanley, Progress in Mathematics 161, Birkhäuser, Boston, 1998. MR 99f:05006 Zbl 0917.52013

[Stanley 1999] R. P. Stanley, Enumerative combinatorics, vol. 2, Cambridge Studies in Advanced Mathematics 62, Cambridge University Press, Cambridge, 1999. MR 2000k:05026 Zbl 0928.05001

[Stanley 2004] R. P. Stanley, "An introduction to hyperplane arrangements", 2004, Available at http://www-math.mit.edu/ rstan/arr.html.

[Tutte 1954] W. T. Tutte, "A contribution to the theory of chromatic polynomials", Canadian J. Math. 6 (1954), 80-91. MR 15,814c Zbl 0055.17101

[Welsh and Whittle 1999] D. J. A. Welsh and G. P. Whittle, "Arrangements, channel assignments, and associated polynomials”, Adv. in Appl. Math. 23:4 (1999), 375-406. MR 2000m:05055 Zbl 0942.05063

[Zaslavsky 1975] T. Zaslavsky, Facing up to arrangements: face-count formulas for partitions of space by hyperplanes, Mem. Amer. Math. Soc. 154, Amer. Math. Soc., Providence, RI, 1975. MR 50 \#9603 Zbl 0296.50010

[Zaslavsky 1995] T. Zaslavsky, "Biased graphs. III. Chromatic and dichromatic invariants", J. Combin. Theory Ser. B 64:1 (1995), 17-88. MR 96g:05139 Zbl 0857.05088

Received September 10, 2004.

\author{
FEDERICO ARDILA \\ MATHEMATICS DEPARTMENT \\ SAN FranCISCO StATE UNIVERSITY \\ 1600 Holloway AVEnue \\ SAN FRANCISCO, CA 94110 \\ UNITED STATES \\ federico@math.sfsu.edu \\ http://math.sfsu.edu/federico
}

